\title{
MicroRNA-124 regulates the radiosensitivity of non-small cell lung cancer cells by targeting TXNRD1
}

\author{
CHUNCHENG HAO $^{1}$, XIANGYING XU $^{1,2}$, JIA MA $^{3}$, JUN XIA $^{3}$, BINGBINGDAI $^{4}$, LILI LIU $^{1}$ and YUYAN MA ${ }^{2}$ \\ ${ }^{1}$ Department of Radiation Oncology, Cancer Hospital of Harbin Medical University; \\ ${ }^{2}$ Institute of Cancer Prevention and Treatment of Heilongjiang, Harbin, Heilongjiang 150040; \\ ${ }^{3}$ Department of Biochemistry and Molecular Biology, Bengbu Medical College, Bengbu, Anhui 233003, P.R. China; \\ ${ }^{4}$ Department of Thoracic and Cardiovascular Surgery, \\ The University of Texas MD Anderson Cancer Center, Houston, TX 77030, USA
}

Received January 28, 2016; Accepted November 17, 2016

DOI: 10.3892/ol.2017.5701

\begin{abstract}
Radiation treatment remains one of the major modalities in the treatment of lung cancer. Although the majority of patients initially respond to treatment with radiation, resistance inevitably develops and leads to treatment failure. Therefore, the identification of the underlying molecular mechanisms of radiation resistance may facilitate the development of novel approaches for overcoming resistance, and enhance the efficacy of treatment with radiation in lung and other types of cancer. In the present study we established three radiation-resistant sub-cell lines derived from the radiation-sensitive lung cancer cell line HCC827. Using a polymerase chain reaction microRNA (miRNA) array, multiple miRNAs were identified to be markedly downregulated in radiation-resistant cells, including miRNA (miR)-124, miR-191 and miR-205. It was observed that overexpression of miR-124 sensitized the resistant cells to treatment with radiation and that thioredoxin reductase 1 (TXNRD1) is a novel target of miR-124. Furthermore, it was demonstrated that knockdown of TXNRD1 using small interfering RNA increased the basal level of reactive oxygen species and sensitized the cells to radiation treatment. The results of the present study demonstrated that multiple miRNAs are downregulated in radiation-resistant lung cancer cells and that downregulation of miR-124 mediates radiation resistance through the targeting of TXNRD1 mRNA expression. The present study revealed a novel molecular mechanism of miRNA-mediated radiation resistance and identified miR-124-regulated TXNRD1 as a novel therapeutic target for overcoming radiation resistance in the treatment of lung cancer.
\end{abstract}

Correspondence to: Dr Xiangying Xu, Department of Radiation Oncology, Cancer Hospital of Harbin Medical University, 150 Haping Road, Harbin, Heilongjiang 150040, P.R. China E-mail: xuxxyy@163.com

Key words: non-small cell lung cancer, radiation resistance, microRNA-124, thioredoxin reductase 1 , reactive oxygen species

\section{Introduction}

Lung cancer occurs as non-small cell lung cancer (NSCLC) in $\sim 85 \%$ of cases and remains the leading cause of cancer-associated mortality. Currently, radiation and chemotherapies are the major modalities for the treatment of lung cancer (1). However, resistance to radiation treatment inevitably develops and leads to treatment failure (2). Understanding the comprehensive underlying mechanism of radiation resistance may facilitate the design of novel approaches, and enhance the efficacy of treatment with radiation and prolong patient survival (3). A previous study has revealed that cancer cell stem cells, a hypoxic environment, the DNA double strand break repair pathway and cell survival pathways are involved in radiation resistance; however, the approaches that can effectively reduce radiation resistance remain to be developed (4).

A recent study has suggested that microRNA (miRNA) expression is significantly altered in radiation-treated cancer cells and that these dysregulated miRNAs modulate radiation sensitivity through the regulation of multiple cellular signaling networks (5). As each miRNA has a number of potential targets, identifying the miRNAs that serve critical roles in regulating radiation sensitivity may facilitate the development of miRNA-mediated therapeutic approaches to overcome radiation resistance. The results from a recent clinical trial of nanoparticle-mediated delivery of miRNA (miR)-200c exhibited an increased efficacy in the use of radiation treatment in lung cancer (6). In addition, identification of the underlying mechanism of miRNA-mediated radiation resistance may enable the development of therapeutic approaches to overcome radiation resistance through the direct targeting of miRNA-regulated downstream targets.

In the present study, novel molecular mechanisms of radiation resistance in lung cancer were revealed using a radiation-resistance model and miRNA gene expression profiling. The results of the present study suggest that downregulation of miRNA-124 (miR-124) mediates radiation resistance in lung cancer cells through the upregulation of thioredoxin reductase 1 (TXNRD1). The present study may provide a rationale for the targeting of antioxidant signaling pathways to enhance the efficacy of radiation therapy in lung cancer treatment. 


\section{Materials and methods}

Cell lines and cell culture. The lung cancer cell lines HCC827 and Calu3 were purchased from American Type Culture Collection (Manassas, VA, USA) and cultured in RPMI 1640 medium (Thermo Fisher Scientific, Inc., Waltham, MA, USA) supplemented with $10 \%$ fetal bovine serum (Thermo Fisher Scientific, Inc.) and penicillin (100 U/ml)/streptomycin $(100 \mu \mathrm{g} / \mathrm{ml})$. All cell cultures were maintained in a humidified $37^{\circ} \mathrm{C}$ incubator with $5 \% \mathrm{CO}_{2}$.

Establishment of radiation-resistant HCC827 lung cancer cells. HCC 827 cells were plated in three $6 \mathrm{~cm}$ plates at a density of $2 \times 10^{6}$ cells/plate. Cells were irradiated with 2 Gy/day $\gamma$-radiation for 10 days (total, $20 \mathrm{~Gy}$ ). After 7 days of recovery, cells were given another two courses of continuous treatment with $2 \mathrm{~Gy} / \mathrm{day} \gamma$-irradiation for 10 days. Cells were allowed to grow at $37^{\circ} \mathrm{C}$ for 2 weeks until clear colonies had formed. Radiation-resistant cell colonies from each plate were pooled for subsequent analysis. These sub-cell lines were named HCC827-irradiation (IR)-clone (C) 1, HCC827-IR-C2 and HCC827-IR-C3.

Clonogenic assay. Between 100 and 800 parental or radiation-resistant cells were seeded in $6 \mathrm{~cm}$ plates. After $24 \mathrm{~h}$, cells were irradiated with $0,2,4,6$ or 8 Gy $\gamma$-irradiation. Cells were then allowed to grow in the incubator for 2 weeks. At the end of the experiment, cells were stained with crystal violet for $10 \mathrm{~min}$ at room temperature and colonies with $>50$ cells were counted using ImageJ (version 1.44a; National Institutes of Health, Bethesda, MD, USA). The survival fraction of the cells was calculated by normalizing the plating efficiency of treated cells by that of control cells as described previously (7).

Cell viability assay. Cells were plated in 96-well plates at a density of $5 \times 10^{3}$ cells/well and, after $24 \mathrm{~h}$, were irradiated with $0,2,4,6$ or 8 Gy $\gamma$-irradiation. Cells were allowed to grow for a further $96 \mathrm{~h}$ and cell viability was analyzed using the MTT (Sigma-Aldrich, Darmstadt, Germany) assay, according to the manufacturer's protocol. Relative cell viabilities of irradiated cells were calculated by normalizing the absorbance at $570 \mathrm{~nm}$ of irradiated cells to that of control cells.

miRNA polymerase chain reaction (PCR) array and validation. The 96-well Human Cancer miRNA PCR Array was purchased from Qiagen, Inc. (Valencia, CA, USA; cat. no. MAH-102A). The array included 88 of the most highly conserved human cancer-associated miRNAs. Total RNA was isolated using TRIzol ${ }^{\circledast}$ reagent (Invitrogen; Thermo Fisher Scientific, Inc.) from HCC827 and the three HCC827 radiation-resistant sub-cell lines, HCC827-IR-C1, HCC827-IR-C2 and HCC827-IR-C3. The assay was performed according to the manufacturer's protocol. A $500 \mathrm{ng}$ sample of total RNA was used for reverse transcription using the RT2 First Strand kit (Qiagen, Inc.). The cDNA was diluted 200-fold and mixed with 2X PCR buffer kit (QuantiTect SYBR Green PCR Master mix) containing polymerase and SYBR Green dye (Qiagen, Inc.). Aliquots of $10 \mu 1$ of diluted cDNA were added to each well of the 96-well miRNA array plate. The PCR was run on a CFX96 RT-PCR detection system (Bio-Rad
Laboratories, Inc., Hercules, CA, USA) and the result was analyzed using $\mathrm{Cq}$ values. The fold changes of miRNA in resistant cells compared with in parental HCC827 cells were calculated for each miRNA by inputting the $\mathrm{Cq}$ values into the manufacturer's web-based software (pcrdataanalysis. sabiosciences.com/mirna/arrayanalysis.php). Individual primers for miR-124 (cat. no. MS00006622), miR-191 (cat. no. MS00003682), miR-205 (cat. no. MS00003780) and small nucleolar RNA C/D box 6 control (cat. no. MS00033705) were purchased from Qiagen, Inc. Aliquots of $10 \mu \mathrm{l}$ of diluted cDNA were used for reverse transcription-quantitative PCR (RT-qPCR) assay using the SYBR Green method. The thermocycling conditions were as follows: $95^{\circ} \mathrm{C}$ for $10 \mathrm{~min}$; followed by 35 cycles of $94^{\circ} \mathrm{C}$ for $10 \mathrm{sec}, 60^{\circ} \mathrm{C}$ for $30 \mathrm{sec}$ and $72^{\circ} \mathrm{C}$ for $45 \mathrm{sec}$. The plate was run as described above and fold changes in miRNA expression were calculated using the $2^{-\Delta \Delta \mathrm{Cq}}$ method (8).

Establishment of stable cell lines using lentiviral transfection. miR-124 (Lenti-miR-124; cat. no. PMIRH-124aPA-1) and miRNA control (Lenti-control; cat. no. PMIRH-000PA-1) plasmids were purchased from System Biosciences, Inc. (Palo Alto, CA, USA). HEK-293T cells were used to generate packaged lentiviruses using a pPACKH1 ${ }^{\mathrm{TM}}$ Lentivector Packaging kit (System Biosciences, Inc.; cat. no. LV500A-1), according to the manufacturer's protocol. For the lentiviral infection, $3 \times 10^{5}$ cells were plated in 6 -well plates, and after $24 \mathrm{~h}$ medium was replaced with $2 \mathrm{ml}$ virus-containing medium and $8 \mu \mathrm{g} / \mathrm{ml}$ Polybrene ${ }^{\circledR}$ (Invitrogen; Thermo Fisher Scientific, Inc.). Cell medium was replaced with fresh medium containing $1 \mu \mathrm{g} / \mathrm{ml}$ puromycin every $48 \mathrm{~h}$ until the cell density reached $90 \%$ confluence. Cells were trypsinized for $5 \mathrm{~min}$ at $37^{\circ} \mathrm{C}$ and split for subsequent analysis.

Apoptosis assay. Cells $\left(2 \times 10^{6}\right)$ were treated with different doses of radiation in a $6 \mathrm{~cm}$ plate, and $48 \mathrm{~h}$ following treatment cells were trypsinized for $5 \mathrm{~min}$ at $37^{\circ} \mathrm{C}$ and washed with PBS twice, followed by centrifugation at $1000 \mathrm{x} g$ at room temperature for $5 \mathrm{~min}$. Cells were stained with propidium iodide/annexin V using a kit (FITC Annexin V Apoptosis Detection kit I) from BD Biosciences (San Diego, CA, USA), according to the manufacturer's protocol. Apoptotic cells were analyzed by flow cytometry and data was analyzed using FlowJo (version 10; Tree Star, Inc., Ashland, OR, USA).

Transfection of small interfering RNA (siRNA) or anti-miRNA. TXNRD1 siRNA (TXNRD1-siRNA-1, GCA CACGCTGTACTGAGGTAA and TXNRD1-siRNA-2, GGAAGAACATGGCATCAAGTT) were purchased from Qiagen, Inc. Lipofectamine ${ }^{\circledR} 2000$ and anti-miRNA-124 (cat. no. AM17000) were purchased from Thermo Fisher Scientific, Inc., and were used for the transfection of TXNRD1 siRNA and anti-miRNA antagomir, according to the manufacturer's protocol. A total of $48 \mathrm{~h}$ following transfection, cells were harvested for western blot analysis, luciferase reporter assays or treatment with radiation for clonogenic assays.

Luciferase Reporter Assay. Luciferase activity was analyzed using a Dual-Luciferase ${ }^{\circledR}$ Reporter Assay kit (Promega Corporation, Madison, WI, USA) following the manufac- 
turer's protocol. The TXNRD1-3'UTR-Luciferase plasmid (cat.no.HUT10621) was purchased from Sigma-Aldrich(Merck Millipore) and the Renilla luciferase control reporter vector pRL-TK was purchased from Promega Corporation. A total of $1 \times 10^{5}$ cells were transfected with $0.5 \mu \mathrm{g}$ TXNRD1-3'-UTR and $0.1 \mathrm{ng}$ pRL-TK using Lipofectamine 2000, according to the manufacturer's protocol. A total of $48 \mathrm{~h}$ following transfection, luciferase activity was measured using the Dual-Luciferase Reporter Assay kit, according to the manufacturer's protocol.

Reactive oxygen species (ROS) analysis. Treated or untreated cells were trypsinized and washed with PBS, and $1 \times 10^{6}$ cells were subsequently resuspended in Hanks' balanced salt solution containing $10 \mu \mathrm{mol} 12^{\prime}, 7^{\prime}$-dichlorodihydrofluorescein diacetate (both Invitrogen; Thermo Fisher Scientific, Inc.). Cells were incubated at $37^{\circ} \mathrm{C}$ for $45 \mathrm{~min}$ followed by washing with PBS twice. Fluorescence intensity was analyzed by flow cytometry as described above.

Western blotting. Antibody targeting TXNRD1 (cat. no. ab16840) was purchased from Abcam (Cambridge, UK), and poly (ADP-ribose) polymerase (PARP; cat. no. 9532s), cleaved-PARP (cat. no. 9542) and apoptosis regulator Bax (cat. no. 5023), were from Cell Signaling Technology, Inc. (Danvers, MA, USA). Anti- nuclear factor erythroid 2-related factor 2 (NRF2) antibody (cat. no. sc-81342) was purchased from Santa Cruz Biotechnology (Dallas, TX. USA). Anti- $\beta$-actin antibody (cat. no. MABT825) was purchased from Sigma-Aldrich (Merck Millipore). Fluorescence-labeled secondary antibody (IRDye ${ }^{\circledR}$ 800CW; cat. no. 925-32211) was purchased from LI-COR Biosciences (Lincoln, NE, USA). Protein lysates were prepared with radioimmunoprecipitation assay buffer and quantified using the Bradford method with a Bradford Protein Assay kit purchased from Bio-Rad Laboratories, Inc. A total of $\sim 40 \mu \mathrm{g}$ total protein was used for western blot analysis. Proteins were separated using SDS-PAGE on a $10 \%$ gel and subsequently transferred to a polyvinylidene fluoride membrane. Membranes were incubated with primary antibodies at $4^{\circ} \mathrm{C}$ overnight and with secondary antibodies at room temperature for $2 \mathrm{~h}$. Protein band images were captured using an Odyssey Imaging scanner from LI-COR Biosciences.

Statistical analysis. Values are presented as the mean \pm standard deviation. The results were analyzed and individual group means were compared using the Student's unpaired $t$-test. Statistical analysis was performed using the GraphPad Prism software (version 6; GraphPad Software, Inc., Lo Jolla, CA. USA). $\mathrm{P}<0.05$ was considered to indicate a statistically significant difference.

\section{Results}

Generation of radiation-resistant lung cancer cells. The human NSCLC cell line HCC827 was used to generate radiation-resistant sub-cell lines. This cell line has been reported previously to be sensitive to radiation as it harbors an epidermal growth factor receptor mutation (9). Radiation-resistant sub-cell lines were established following three courses of continuous irradiation at a dose of 2 Gy/day for 10 days (total,
60 Gy). Resistant colonies were formed after 2 weeks of recovery without treatment (Fig. 1A). All colonies from the same plate were pooled. In total, three resistant sub-cell lines, HCC827-IR-C1, HCC827-IR-C2 and HCC827-IR-C3, were established. Radiation resistance of all three sub-cell lines was further confirmed by clonogenic survival (Fig. 1B) and cell viability (Fig. 1C) assays.

miRNA profiling identified downregulation of miRNA expression in radiation-resistant cells. Using a PCR-based miRNA array focusing on 88 human cancer-associated miRNAs in the format of a 96-well plate, it was observed that a panel of miRNAs, including miR-124, miR-191, miR-205, miR-128 and miR-34c, were significantly downregulated in radiation-resistant HCC827 cells compared with parental cells $(\mathrm{P}<0.01$; Fig. 2A). Three of the downregulated miRNAs, miR-124, miR-191 and miR-205, were selected for further validation using RT-qPCR. Consistent with the PCR array results, miR-124, miR-191 and miR-205 were significantly downregulated in all three radiation-resistant sub-cell lines compared with the parental cell line $(\mathrm{P}<0.01$; Fig. $2 \mathrm{~B})$.

Overexpression of miR-124 sensitizes radiation-resistant lung cancer cells to radiation treatment. As miR-124 has been reported previously to serve an important role in cancer (10-13), it was selected for further investigation, despite its role and underlying mechanism behind lung cancer radiosensitivity remaining unclear. To validate whether ectopic expression of miR-124 reversed the radiation resistance in radiation-resistant cells, two stable cell lines were established using lentivirus-mediated overexpression of a precursor of miR-124. Expression of miR-124 in the stable cells was confirmed using RT-qPCR (Fig. 3A). A representative image of the clonogenic assay is shown in Fig. 3B. The clonogenic assay demonstrated that ectopic overexpression of miR-124 significantly decreased the survival fraction of HCC827-IR-C1 and HCC827-IR-C2 cells following treatment with increasing doses of radiation $(\mathrm{P}<0.01$; Fig. $3 \mathrm{C})$. Analysis of apoptosis indicated that, compared with cells transfected with the control siRNA, re-expression of miR-124 induced increased apoptosis in radiation-resistant cells treated with 2 and 4 Gy $\gamma$-irradiation (Fig. 3D), which was further confirmed using western blot analysis to evaluate cleavage of PARP, a cell apoptosis marker (Fig. 3E). These results suggest that ectopic expression of miR-124 sensitizes radiation-resistant cells to treatment with radiation.

miR-124 regulates TXNRD1 expression. To further investigate the mechanism of miR-124-mediated radiosensitization, potential target genes of miR-124 were analyzed using TargetScan online software (version 6.0; Whitehead Institute for Biomedical Research, Cambridge, MA, USA). It was observed that one of the potential targets was TXNRD1. The TXRND1 gene encodes a protein that is able to protect cells from oxidative stress by reducing thioredoxin (TXN) levels. There is a conserved binding site for miR-124 on the 3'-untranslated region (UTR) of mRNA (Fig. 4A and B). Using western blotting it was confirmed that the protein expression of TXNRD1 was increased in radiation-resistant cells compared with that in parental HCC827 cells; however, the transcription 

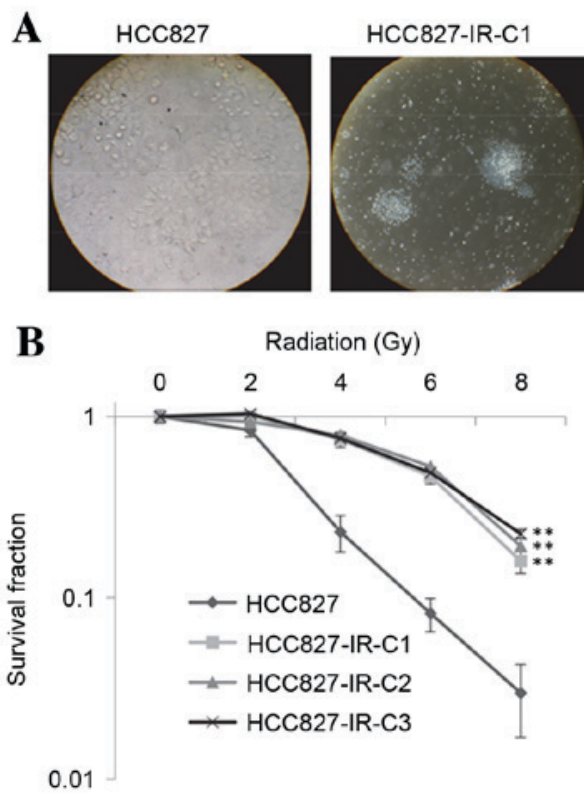

HCC827-IR-C2

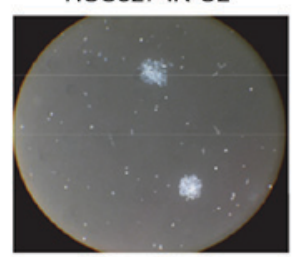

C



Figure 1. Establishment of radiation-resistant lung cancer cells. (A) Representative images of three independent radiation-resistant sub-cell lines derived from HCC827 cells. Radiation-resistant colonies were established following three continuous courses of irradiation (total, 60 Gy). (B) Clonogenic assays were performed to analyze the responses of radiation-resistant cells and parental HCC827 cells to irradiation at the indicated doses. (C) Cell viability assay (MTT) of radiation-resistant and parental cells following irradiation. Values are presented as the mean \pm standard deviation $(\mathrm{n}=3) .{ }^{* *} \mathrm{P}<0.01 \mathrm{vs}$. the parental cells.

$\mathbf{A}$

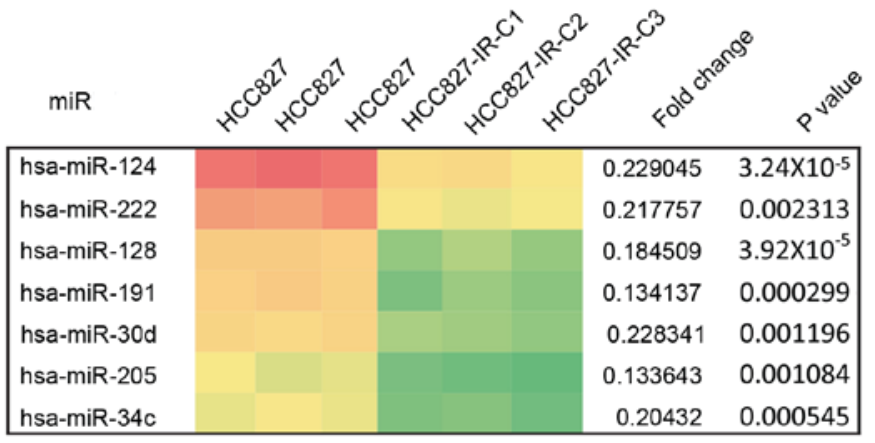

B
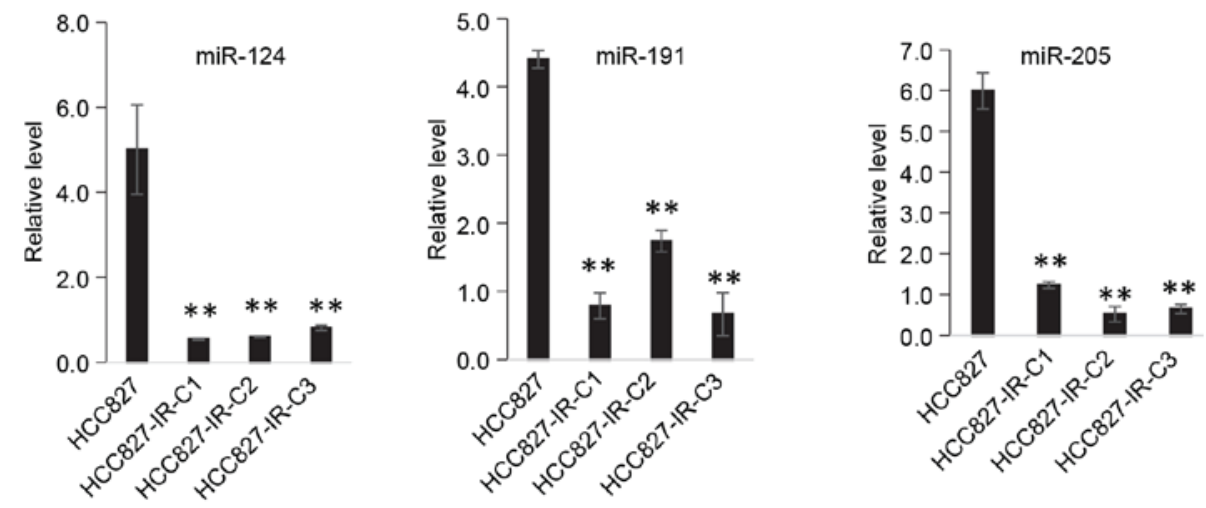

Figure 2. Radiation-resistant cells express decreased levels of various miRNAs. (A) An miR PCR array was used to analyze differential miR expression in parental HCC827 and radiation-resistant cells, and a heat map of miR expression was generated to visualize the most significantly downregulated miRs in radiation-resistant cells. (B) Validation of the three downregulated miRs, miR-124, miR-191 and miR-205, using reverse transcription-quantitative PCR. SNORD6 was used as an internal control and the values are relative to the parental cells. ${ }^{* *} \mathrm{P}<0.01$ vs. the parental cells. hsa, Homo sapiens; miR, microRNA; PCR, polymerase chain reaction.

factor NRF2, which regulates multiple antioxidant molecules, including TXNRD1, was not upregulated in radiation-resistant cells (Fig. 4C). In addition, overexpression of miR-124 reduced the expression of TXNRD1 in radiation-resistant cells (Fig. 4D). By contrast, inhibition of miR-124 using an antagomir upregulated TXNRD1 protein expression in 




B

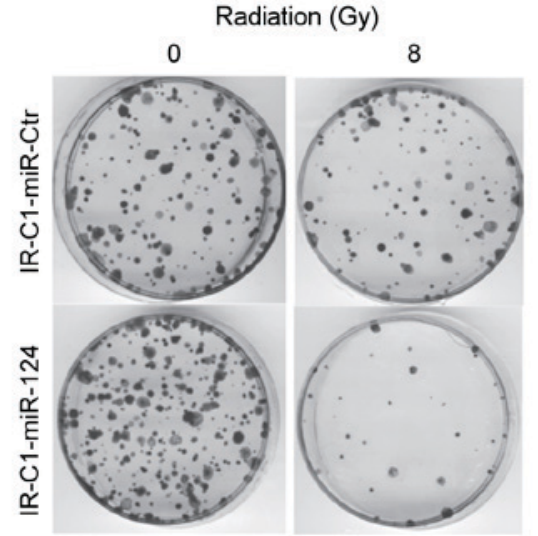

C

Radiation (Gy)
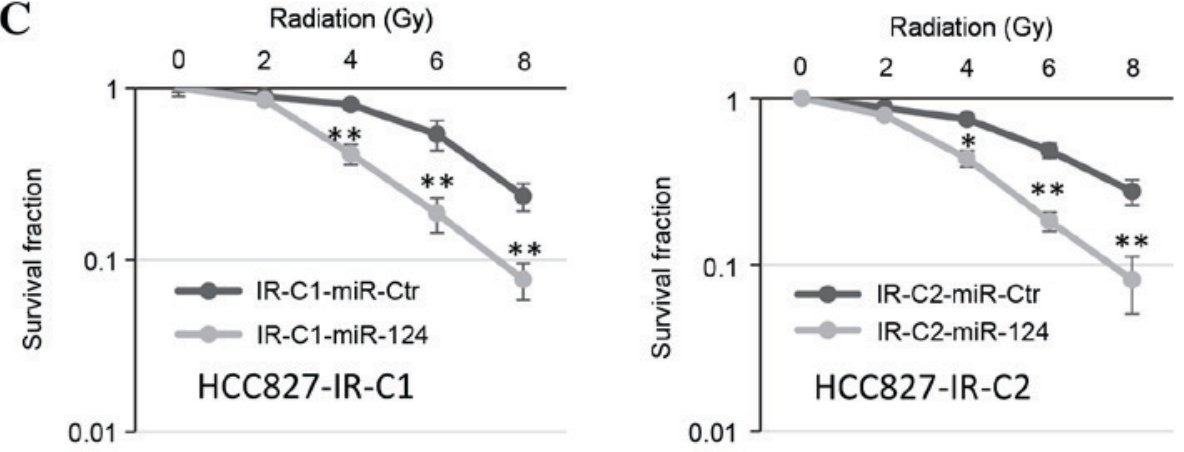

D

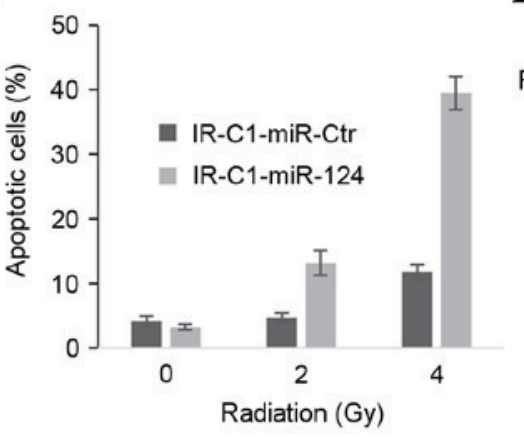

$\mathbf{E}$

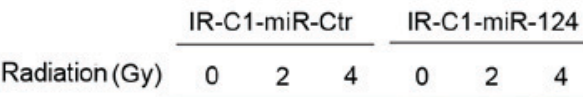

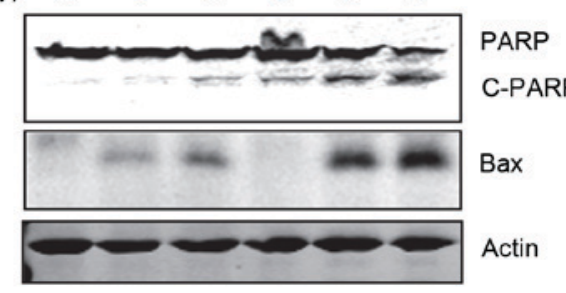

Figure 3. Overexpression of miR-124 sensitizes radiation-resistant cells to radiation. (A) Cells with stable overexpression of miR-124 were established using lentivirus-mediated transduction. (B) Representative images of the clonogenic assay using IR-C1 cells. Compared with the control miR-transfected cells, miR-124 reduced IR-C1 cell survival following treatment with 8 Gy radiation. (C) The clonogenic assay was performed, and the survival fractions of the cells treated with increasing doses of radiation were analyzed by calculating the cell plating efficiency. (D) Cells transduced with Ctr or miR-124 were treated with 2 or $4 \mathrm{~Gy}$ radiation, and $48 \mathrm{~h}$ following treatment, cells were harvested for propidium iodide/annexin V staining of apoptotic cells. (E) Cells were treated with the indicated doses of radiation, and $48 \mathrm{~h}$ following treatment, cell lysates were collected for western blot analysis. Values are presented as the mean \pm standard deviation $(\mathrm{n}=3)$. ${ }^{*} \mathrm{P}<0.05 ;{ }^{* *} \mathrm{P}<0.01$ vs. miR-Ctr. Bax, apoptosis regulator Bax; C-PARP, cleaved poly (ADP-ribose) polymerase; IR-C1, HCC827-irradiation-clone 1 cells; miR, microRNA; Ctr, control miR.

HCC827 and Calu3 cells (Fig. 4E). Using a 3'-UTR luciferase reporter of TXNRD1, it was further demonstrated that inhibition of miR-124 increased luciferase activity in HCC 827 and Calu3 cells (Fig. 4F). These results demonstrate that TXNRD1 is a target of miR-124 in lung cancer cells.

Inhibition of TXNRD1 sensitizes radiation-resistant cells to irradiation. To further investigate whether miR-124 mediates radiosensitivity by targeting TXNRD1, two distinct TXNRD1 siRNAs were used to knock down protein expression of TXNRD1 in HCC827-IR-C1 and HCC827-IR-C2 cells, and the subsequent effects were analyzed using clonogenic and cell viability assays. Using western blotting, the inhibitory efficiency of two TXNRD1 siRNAs, siRNA-1 and siRNA-2 were initially evaluated. It was observed that siRNA-2 more effectively suppressed TXNRD1 expression in the two radiation-resistant sub-cell lines (Fig. 5A). As TXNRD1 is an important antioxidant molecule, inhibition of TXNRD1 is expected to increase cellular ROS levels. Treatment with siRNA-1 and siRNA-2 increased the basal level of ROS in radiation-resistant cells, with siRNA-2 increasing ROS levels more compared with siRNA-1 (Fig. 5B). siRNA-2 was therefore selected for subsequent experiments. Compared with the control siRNA (siRNA-C), transfection with siRNA-2 decreased the survival fraction of HCC827-IR-C1 and HCC827-IR-C2 cells following treatment with radiation (Fig. 5C). Western blotting further confirmed that knockdown of TXNRD1 combined with treatment with radiation induced 
A

$$
\begin{array}{ll}
\text { Position 2059-2065 of TXNRD1 3' UTR } & 5^{\prime} \\
\text { hsa-miR-124-3P.1 } & \text { 3' UAAUAAUAUCCACGUGUGCCUUC... }
\end{array}
$$
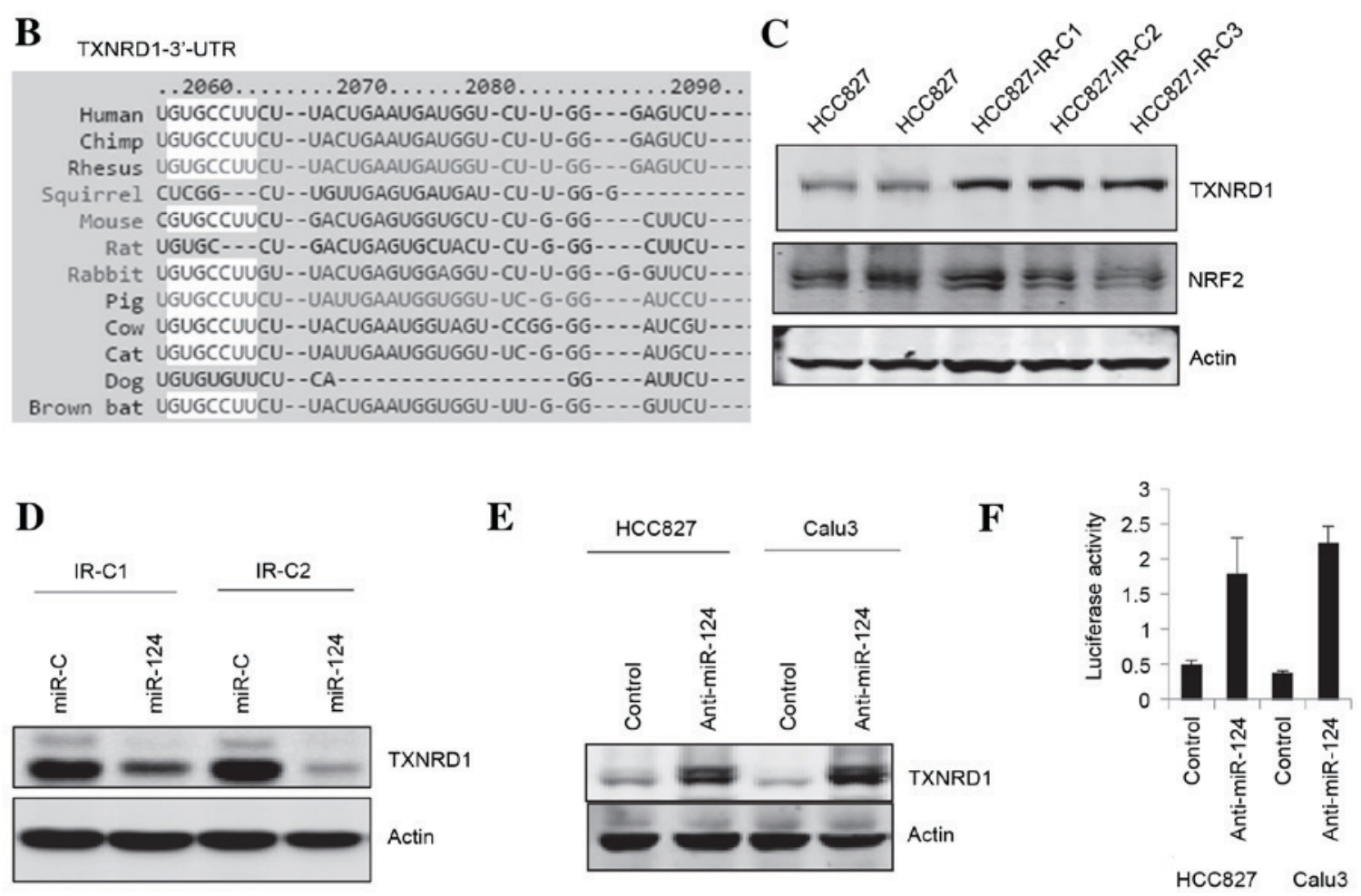

Figure 4. miR-124 regulates TXNRD1 protein expression in lung cancer cells. (A) A putative miR-124-binding site on the 3'-UTR of the TXNRD1 mR is shown. (B) The binding sites for miR-124 on the 3'-UTR of TXNRD1 are conserved across different species. (C) Cell lysates from the parental and the three radiation-resistant cell lines were used for western blot analysis with the indicated antibodies. (D) miR-C or miR-124 stably transfected radiation-resistant cells were examined for TXNRD1 expression using western blotting. (E) HCC827 and Calu3 cells were transfected with anti-miR control or anti-miR-124, and $48 \mathrm{~h}$ following transfection, cell lysates were harvested for western blot analysis. (F) Luciferase reporter assay. HCC827 and Calu3 cells were transfected with anti-miR control or anti-miR-124, and $48 \mathrm{~h}$ following transfection, cells were further transfected with TXNRD1-3'-Luc and pRL-TK for $24 \mathrm{~h}$. hsa, Homo sapiens; IR-C1, HCC827-IR-C1 cells; 3'-Luc, 3'-luciferase; miR, microRNA; miR-C, control miRNA; NRF2, nuclear factor erythroid 2-related factor 2; pRL-TK, Renilla luciferase vector with thymidine kinase promoter; TXNRD1, thioredoxin reductase 1; UTR, untranslated region.

PARP cleavage in HCC827-IR-C1 and in HCC827-IR-C2 cells (Fig. 5D). These results suggest that miR-124-regulated radiation sensitivity acts by targeting TXNRD1.

\section{Discussion}

In the present study, a cell line model of lung cancer radiation resistance was developed and the miRNA-mediated molecular mechanism of radiation resistance was investigated. miR-124 was identified to be significantly downregulated in radiation-resistant sub-cell lines. Other downregulated miRNAs included miR-191 and miR-205, which have been reported previously to be involved in radiation resistance in lung and breast cancer, respectively $(14,15)$. It was demonstrated that overexpression of miR-124 was able to sensitize radiation-resistant cells to radiation by decreasing the cell survival fraction and viability, and identified that TXNRD1 is an important target of miR-124. Knockdown of TNXRD1 with specific siRNA increased basal level of ROS production and sensitized the cells to radiation treatment.
Previous studies have demonstrated that downregulation of miR-124 is associated with metastasis and poor prognosis in lung and breast cancer $(13,16)$. Molecules involved in epithelial-mesenchymal transition, including Slug and signal transducer and activator of transcription 3 have been demonstrated to be targets of miR-124 $(10,16)$. miR-124 was also demonstrated to be able to suppress lung cancer metastasis through the regulation of myosin $\mathrm{X}$ expression (12). A recent study also indicated that miR-124 sensitizes colorectal and glioma cells to radiation by targeting paired related homeobox 1 and cyclin-dependent kinase 4, respectively $(17,18)$. In the present study, it was observed that miR-124 was downregulated in radiation-resistant lung cancer cells, and overexpression of miR-124 sensitized lung cancer cells to radiation, which is consistent with previous findings in other types of cancer $(17,18)$. In addition, it was observed that TXNRD1 is a novel target of miR-124 and is significantly upregulated in radiation-resistant lung cancer cells. The present study suggested that increased expression of TXNRD1 as a result of downregulation of miR-124 is one of the underlying mechanisms of radiation resistance in lung cancer. 
A
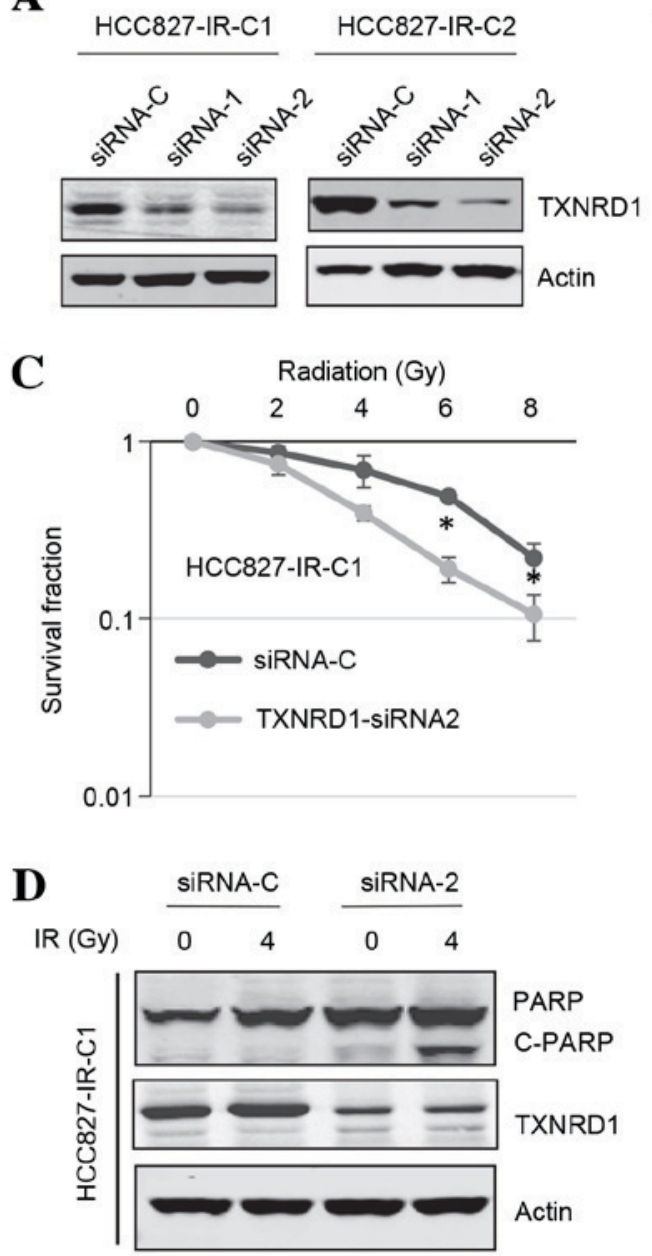

B
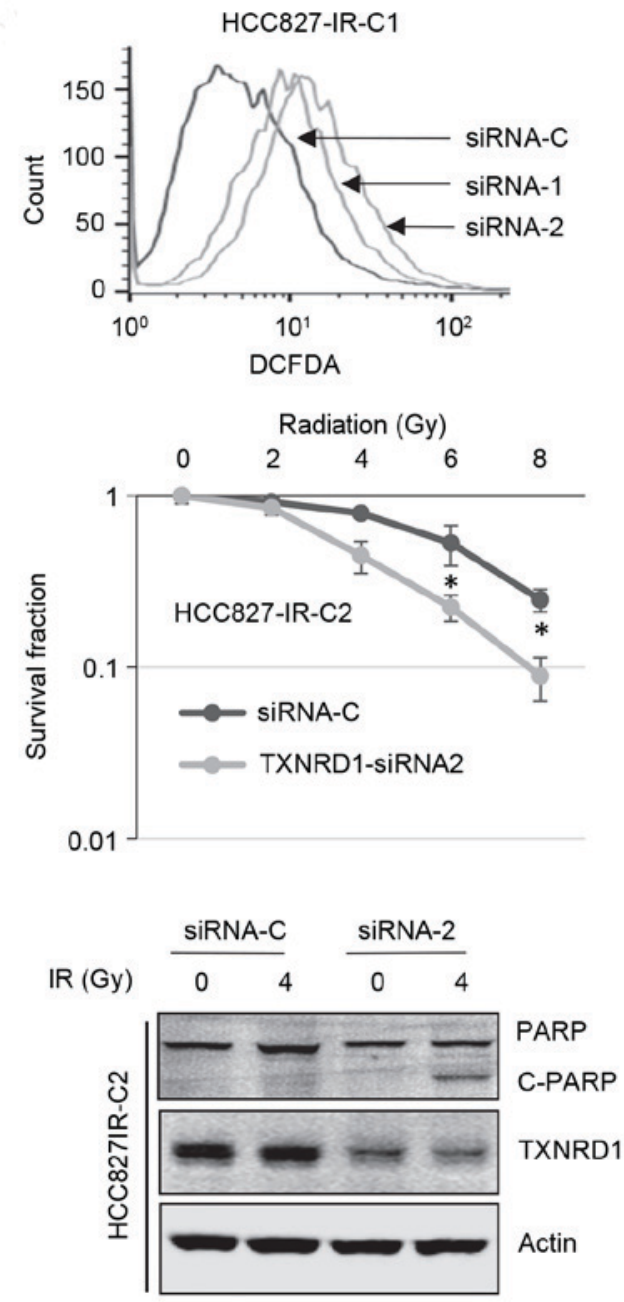

Figure 5. Knockdown of TXNRD1 with siRNA sensitizes the radiation-resistant cells to radiation. (A) Western blotting was performed to evaluate the inhibition of TXNRD1 using two distinct siRNAs in two radiation-resistant sub-cell lines. (B) Reactive oxygen species assay. HCC827-IR-C1 cells were transfected with TXNRD1-siRNA-2, and $48 \mathrm{~h}$ following transfection, cells were harvested and stained with the DCFDA kit followed by flow cytometric analysis. (C) Clonogenic assay. Cells were transfected with siRNA-C or TXNRD1 siRNA, and $48 \mathrm{~h}$ following transfection, cells were re-seeded into a 6 cm plate for the clonogenic assay with the indicated doses of radiation. Cell survival fractions were calculated using the plating efficiency. Values are presented as mean \pm standard deviation $(\mathrm{n}=3)$. ${ }^{*} \mathrm{P}<0.05 \mathrm{vs}$. siR-C. (D) A total of $48 \mathrm{~h}$ following transfection, cells were treated with the indicated dose of radiation, and then $48 \mathrm{~h}$ following treatment, were harvested for western blot analysis. C, control; C-PARP, cleaved poly (ADP-ribose) polymerase; DCFDA, 2',7'-dichlorofluorescein diacetate; IR, ionizing radiation; ROS, reactive oxygen species; siRNA, short interfering RNA; TXNRD1, thioredoxin reductase 1.

TXNRD1 is one of the major molecules regulating the cellular redox balance by reducing oxidized TXN and serves an important role in protecting cells against oxidative stress (19). Overexpression of TXNRD1 has been reported in many types of cancer, including lung cancer (20-22), and it is involved in the resistance of cancer cells to chemotherapeutics and targeted inhibitors $(23,24)$. The results of the present study demonstrated that direct knockdown of TNXRD1 increased the basal level of ROS, and significantly sensitized radiation-resistant lung cancer cells to radiation. Although the detailed mechanism of TXNRD1-mediated radiation resistance remains to be elucidated, TXNRD1-mediated radiation resistance may involve the regulation of ROS production, as radiation-resistant cancer stem cells have exhibit low levels of ROS due to activation of the free radical scavenger system $(25,26)$.

Ionizing radiation induces DNA double strand breaks, thus miRNAs that regulate DNA damage and repair signaling pathways have been demonstrated to be involved in radiation sensitivity $(27,28)$. miRNAs involved in cancer stem cell self-renewal, autophagy, hypoxia and survival signaling pathways also affect the radiosensitivity of cancer cells $(15,29,30)$. In addition, a recent study indicated that delivery of miR-200c enhanced radiosensitivity in lung cancer by targeting the oxidative-stress-response genes peroxiredoxin 2 and GA-binding protein/nuclear respiratory factor 2 (6). Certain miRNAs may modulate the radiation sensitivity of cancer cells through the regulation of multiple signaling pathways (31). The present study has overlapping observations with those of previous studies, for example, downregulation of miR-191 and miR-205 in radiation-resistant breast cancer cells has been reported $(15,32)$, and downregulation of miR-124 was also reported in radiation-resistant brain tumor cells (18). However, the role of miR-124 in lung cancer radiation sensitivity was not reported in previous models. Furthermore, in the present study, the HCC 827 cell line was used, which has been demonstrated previously to be sensitive to radiation treatment and has not been used in previous radiation-resistance models. In 
addition, the radiation-resistance model in the present study was established using low continuous doses of radiation ( $2 \mathrm{~Gy}$ ) to give a total of $60 \mathrm{~Gy}$, which more closely mimics clinical radiation treatment schedules. Validation of the observations in additional NSCLC cell lines and further investigation of other miR-124 targets involved in radiation resistance will be investigated in future studies.

In conclusion, using an miRNA array it was identified that a panel of miRNAs were downregulated in radiation-resistant NSCLC lung cancer cells and that miR-124-mediated radiation resistance may occur through the regulation of TXNRD1 expression. The results of the present study suggest that modulating miR-124 expression or directly targeting TXNRD1 are novel approaches to enhancing radiation therapy in NSCLC.

\section{Acknowledgements}

The present study was supported by the Heilongjiang Provincial Science and Technology Projects (grant no. WB12C101), and Special Fund for Innovative Talent in Science and Technology Research of Harbin (grant no. 2012RFXXS063).

\section{References}

1. Reck M, Heigener DF, Mok T, Soria JC and Rabe KF: Management of non-small-cell lung cancer: Recent developments. Lancet 382: 709-719, 2013.

2. Yom SS: Accelerated repopulation as a cause of radiation treatment failure in non-small cell lung cancer: Review of current data and future clinical strategies. Semin Radiat Oncol 25: 93-99, 2015

3. Ausborn NL, Le QT, Bradley JD, Choy H, Dicker AP, Saha D, Simko J, Story MD, Torossian A and Lu B: Molecular profiling to optimize treatment in non-small cell lung cancer: A review of potential molecular targets for radiation therapy by the translational research program of the radiation therapy oncology group. Int J Radiat Oncol Biol Phys 83: e453-e464, 2012.

4. Murren JR and Buzaid AC: Chemotherapy and radiation for the treatment of non-small-cell lung cancer. A critical review. Clin Chest Med 14: 161-171, 1993.

5. MacDonagh L, Gray SG, Finn SP, Cuffe S, O'Byrne KJ and Barr MP: The emerging role of microRNAs in resistance to lung cancer treatments. Cancer Treat Rev 41: 160-169, 2015.

6. Cortez MA, Valdecanas D, Zhang X, Zhan Y, Bhardwaj V, Calin GA, Komaki R, Giri DK, Quini CC, Wolfe T, et al: Therapeutic delivery of miR-200c enhances radiosensitivity in lung cancer. Mol Ther 22: 1494-1503, 2014.

7. Franken NA, Rodermond HM, Stap J, Haveman J and van Bree C: Clonogenic assay of cells in vitro. Nat Protoc 1: 2315-2319, 2006

8. Schmittgen TD and Livak KJ: Analyzing real-time PCR data by the comparative C(T) method. Nat Protoc 3: 1101-1108, 2008.

9. Das AK, Sato M, Story MD, Peyton M, Graves R, Redpath S, Girard L, Gazdar AF, Shay JW, Minna JD and Nirodi CS: Non-small-cell lung cancers with kinase domain mutations in the epidermal growth factor receptor are sensitive to ionizing radiation. Cancer Res 66: 9601-9608, 2006.

10. Li X, Yu Z, Li Y, Liu S, Gao C, Hou X, Yao R and Cui L: The tumor suppressor miR-124 inhibits cell proliferation by targeting STAT3 and functions as a prognostic marker for postoperative NSCLC patients. Int J Oncol 46: 798-808, 2015.

11. Patnaik SK, Kannisto E, Knudsen S and Yendamuri S: Evaluation of microRNA expression profiles that may predict recurrence of localized stage I non-small cell lung cancer after surgical resection. Cancer Res 70: 36-45, 2010.

12. Sun Y, Ai X, Shen S and Lu S: NF- $\kappa$ B-mediated miR-124 suppresses metastasis of non-small-cell lung cancer by targeting MYO10. Oncotarget 6: 8244-8254, 2015.
13. Zhang Y, Li H, Han J and Zhang Y: Down-regulation of microRNA-124 is correlated with tumor metastasis and poor prognosis in patients with lung cancer. Int J Clin Exp Pathol 8: 1967-1972, 2015

14. Liu Z and Huang S: Inhibition of miR-191 contributes to radiation-resistance of two lung cancer cell lines by altering autophagy activity. Cancer Cell Int 15: 16, 2015.

15. Zhang P, Wang L, Rodriguez-Aguayo C, Yuan Y, Debeb BG, Chen D, Sun Y, You MJ, Liu Y, Dean DC, et al: miR-205 acts as a tumour radiosensitizer by targeting ZEB1 and Ubc13. Nat Commun 5: 5671, 2014.

16. Liang YJ, Wang QY, Zhou CX, Yin QQ, He M, Yu XT, Cao DX, Chen GQ, He JR and Zhao Q: MiR-124 targets Slug to regulate epithelial-mesenchymal transition and metastasis of breast cancer. Carcinogenesis 34: 713-722, 2013.

17. Zhang Y, Zheng L, Huang J, Gao F, Lin X, He L, Li D, Li Z, Ding Y and Chen L: MiR-124 Radiosensitizes human colorectal cancer cells by targeting PRRX1. PLoS One 9: e93917, 2014.

18. Deng X, Ma L, Wu M, Zhang G, Jin C, Guo Y and Liu R: miR-124 radiosensitizes human glioma cells by targeting CDK4. J Neurooncol 114: 263-274, 2013.

19. Arnér ES: Focus on mammalian thioredoxin reductases-important selenoproteins with versatile functions. Biochim Biophys Acta 1790: 495-526, 2009.

20. Fernandes AP, Capitanio A, Selenius M, Brodin O, Rundlöf AK and Björnstedt M: Expression profiles of thioredoxin family proteins in human lung cancer tissue: Correlation with proliferation and differentiation. Histopathology 55: 313-320, 2009.

21. Cadenas C, Franckenstein D, Schmidt M, Gehrmann M, Hermes M, Geppert B, Schormann W, Maccoux LJ, Schug M, Schumann A, et al: Role of thioredoxin reductase 1 and thioredoxin interacting protein in prognosis of breast cancer. Breast Cancer Res 12: R44, 2010.

22. Iwasawa S, Yamano Y, Takiguchi Y, Tanzawa H, Tatsumi K and Uzawa K: Upregulation of thioredoxin reductase 1 in human oral squamous cell carcinoma. Oncol Rep 25: 637-644, 2011.

23. Nguyen P, Awwad RT, Smart DD, Spitz DR and Gius D: Thioredoxin reductase as a novel molecular target for cancer therapy. Cancer Lett 236: 164-174, 2006.

24. Dai B, Yoo SY, Bartholomeusz G, Graham RA, Majidi M, Yan S, Meng J, Ji L, Coombes K, Minna JD, et al: KEAP1-dependent synthetic lethality induced by AKT and TXNRD1 inhibitors in lung cancer. Cancer Res 73: 5532-5543, 2013.

25. Diehn M, Cho RW, Lobo NA, Kalisky T, Dorie MJ, Kulp AN, Qian D, Lam JS, Ailles LE, Wong M, et al: Association of reactive oxygen species levels and radioresistance in cancer stem cells. Nature 458: 780-783, 2009.

26. Tothova $Z$ and Gilliland DG: A radical bailout strategy for cancer stem cells. Cell Stem Cell 4: 196-197, 2009.

27. Li Y, Han W, Ni TT, Lu L, Huang M, Zhang Y, Cao H, Zhang HQ, Luo W and Li H: Knockdown of microRNA-1323 restores sensitivity to radiation by suppression of PRKDC activity in radiation-resistant lung cancer cells. Oncol Rep 33: 2821-2828, 2015.

28. van Jaarsveld MT, Wouters MD, Boersma AW, Smid M, van Ijcken WF, Mathijssen RH, Hoeijmakers JH, Martens JW, van Laere S, Wiemer EA and Pothof J: DNA damage responsive microRNAs misexpressed in human cancer modulate therapy sensitivity. Mol Oncol 8: 458-468, 2014.

29. Grosso S, Doyen J, Parks SK, Bertero T, Paye A, Cardinaud B, Gounon P, Lacas-Gervais S, Noël A, Pouysségur J, et al: MiR-210 promotes a hypoxic phenotype and increases radioresistance in human lung cancer cell lines. Cell death Dis 4: e544, 2013.

30. Ma Y, Xia H, Liu Y and Li M: Silencing miR-21 sensitizes non-small cell lung cancer A549 cells to ionizing radiation through inhibition of PI3K/Akt. Biomed Res Int 2014: 617868, 2014.

31. Chang JH, Hwang YH, Lee DJ, Kim DH, Park JM, Wu HG and Kim IA: MicroRNA-203 modulates the radiation sensitivity of human malignant glioma cells. Int J Radiat Oncol Biol Phys 94: 412-420, 2016.

32. Yi H, Liang B, Jia J, Liang N, Xu H, Ju G, Ma S and Liu X: Differential roles of miR-199a-5p in radiation-induced autophagy in breast cancer cells. FEBS Lett 587: 436-443, 2013. 